\title{
The Molecular Zeeman Effect of Norbornadiene, its $g$-Values, Magnetizability Anisotropies, and Molecular Electric Quadrupole Moment; A High-Resolution Microwave Fourier-Transform Study Combined with Quantum Chemical Calculations
}

\author{
K. Voges, D. H. Sutter, K. Ruud ${ }^{\mathrm{a}, \mathrm{b}}$, and T. Helgaker ${ }^{\mathrm{b}}$ \\ Christian-Albrechts-Universität, D-24098 Kiel \\ ${ }^{\text {a }}$ Department of Physics and Measurement Technology, Linköping University, S-58183 Linköping \\ ${ }^{b}$ Department of Chemistry, University of Oslo, P. O. Box 1033 Blindern, N-0315 Oslo \\ Z. Naturforsch. 53 a, 67-76 (1998); received January 3, 1998

\begin{abstract}
The molecular Zeeman effect is reported for norbornadiene at fields near $18 \mathrm{kG}$. The experimental
\end{abstract} \\ results are for the molecular $g$-values: $g_{a a}=0.02860(16), g_{b b}=0.05271(11), g_{c c}=0.00142(26)$, \\ for the magnetizability anisotropies: $2 \xi_{a a}-\xi_{b b}-\xi_{c c}=-0.41(26) \cdot 10^{-6} \mathrm{erg} \mathrm{G}^{-2} \mathrm{~mol}^{-1}$, \\ $2 \xi_{b b}-\xi_{c c}-\xi_{a a}=+40.72(27) \cdot 10^{-6} \mathrm{erg} \mathrm{G}^{-2} \mathrm{~mol}^{-1}$ and for the molecular electric quadrupole \\ moments: $Q_{a a}=-1.78(20) \cdot 10^{-26}$ esu cm${ }^{2}, Q_{b b}=3.73(20) \cdot 10^{-26} \mathrm{esu} \mathrm{cm}^{2}, Q_{c c}=-1.94(30)$. \\ $10^{-26}$ esu cm${ }^{2}$, with the $c$-axis of the molecular inertia tensor aligned to the $C_{2 \mathrm{v}}$-axis of the equi- \\ librium configuration. The results of $a b$-initio calculations, using London type atomic orbitals as \\ basis functions, are reported for the $g$-tensor, the magnetizability tensor and the molecular electric \\ quadrupole moment tensor and are compared to the experimental findings. The possibilities of \\ strain induced magnetizability exaltations and of systematic differences between gas phase and \\ bulk phase magnetizabilities are discussed.
}

\section{Introduction}

The structure of norbornadiene (bicyclo [2.2.1] hepta-2,5-diene, $\mathrm{C}_{7} \mathrm{H}_{8}$ ), with its interesting ability for photo-sensitized valence isomerisation to quadricyclane, has been determined recently by Bauder and coworkers $[1,2]$. In the following we present the results of a rotational Zeeman effect study. Such a study [3] directly gives the anisotropies in the magnetizability tensor. It also leads to vibronic ground state expectation values for the molecular electric quadrupole moment. The magnetizability anisotropies in turn give information on nonlocal ring currents within the electron cloud, which might be induced by application of an exterior magnetic field (1.c. [3], Chapt. C). Such ring currents are most prominent in delocalized $\pi$ electron systems. In norbornadiene we suspected that strain induced overlap of the $\pi$-orbitals might lead to a magnetizability exaltation along the $c$-axis of the molecule. For the orientation of the principal inertia axes system compare Figure 1.

Reprint requests to Prof. D.-H. Sutter; Fax: +49 431880 1416 / 1704, E-mail: sutter@phc.uni-kiel.de.

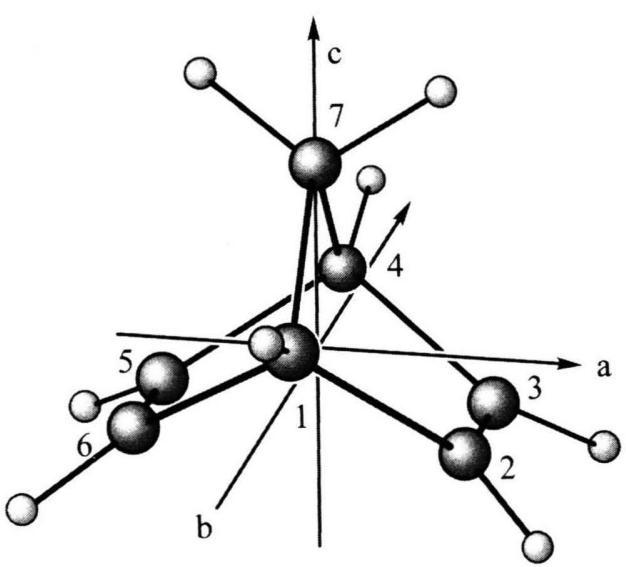

Fig. 1. Norbornadiene with the numbering of the carbon nuclei and the orientation of the principal inertia axes system.

Since the permanent electric dipole moment of norbornadiene is comparatively small $\left(|\mu|=\left|\mu_{c}\right|=\right.$ 0.05866(9) $D,[1])$, conventional Stark-effect modulation microwave spectroscopy would have been inappropriate because of low modulation efficiency. Therefore the technique of pulsed waveguide Fourier

0932-0784 / 98 / 0100-0067 \$06.00 (C) - Verlag der Zeitschrift für Naturforschung, D-72072 Tübingen 

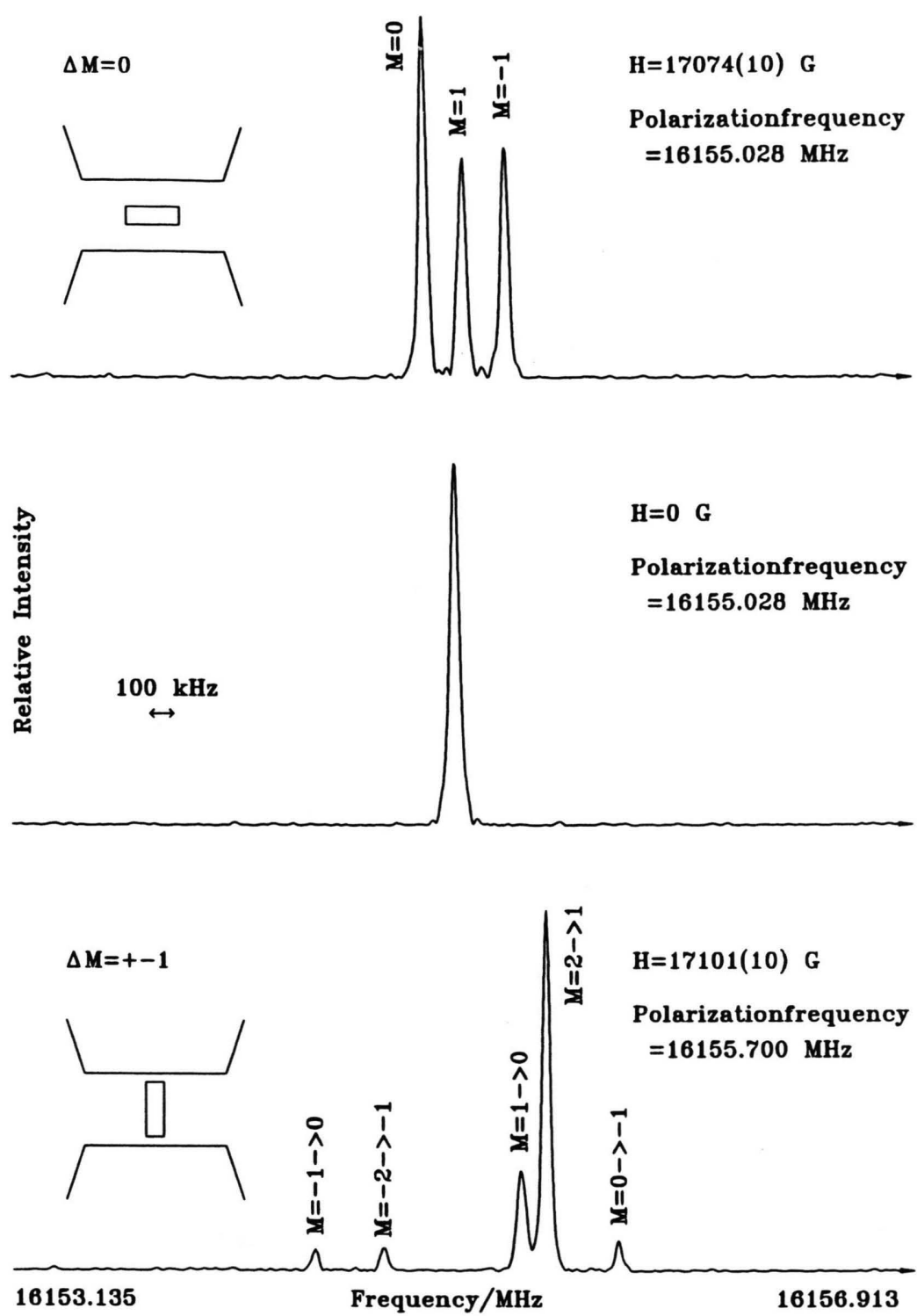

Fig. 2. Fourier transform spectra of the $2(2,0)-1(1,0)$ rotational transition of norbornadiene observed at zero field and at fields of 17074 Gauss $(\Delta M=0)$ and 17101 Gauss $(\Delta M= \pm 1)$ (compare too Table 2 and text). The inserts show the orientation of the waveguide cross section between the pole faces of the magnet. transform microwave spectroscopy was used instead. For a recent report on the field of Fourier transform microwave spectroscopy, see [4].

\section{Experimental}

The sample of norbornadiene was purchased from Aldrich (purity: $99 \%$, b. p. $89^{\circ}$ C). It was used for the spectroscopic studies after several bulb to bulb distillations without further purification.

The Zeeman measurements were carried out with the waveguide Fourier transform microwave spectrometer described earlier [5]. As compared to the original setup, the spectrometer has been improved further. The changes were aimed to reduce the noise level and to eliminate the effects of coherent perturbations as far as possible.

First, the pair of phase locked microwave oscillators (signal oscillator and local oscillator) was replaced by a single microwave source as local oscillator. The polarization signal was derived from the local oscillator frequency. To this end a fraction of the local oscillator output was periodically fed into a single side band mixer. A $160 \mathrm{MHz} \mathrm{cw}$ signal, derived from the master clock of the system, was fed into the second input of the mixer. The upper side band 
from the mixer output, $\nu_{\text {signal }}=\nu_{\text {local }}+160 \mathrm{MHz}$, was fed through a computer controlled YIG-filter to a two stage microwave amplifier chain to provide the polarization signal. As second stage a Hughes $8010 \mathrm{H}$ traveling wave tube amplifier was used (maximum output power: $15 \mathrm{~W}$ ). Since the microwave path to the mixer was opened only for pulse formation, no signal frequency radiation was present during the free induction decay periods. This eliminates coherent perturbations caused by signal leakage into the detection system.

Second, an image rejection mixer (MITEQ No.: IR0618LC2C) was used for the down conversion of the free induction decay signals into the $160 \mathrm{MHz}$ band. This reduces the noise level at the detector by a factor of two as compared to the previous setup with a simple waveguide mixer.

Third, for further reduction of coherent perturbations, the feature of base line subtraction was added to the phase alternating pulse sequence technique already used in the original setup. To this end data acquisition was carried out in three-step cycles. In the first step $2^{15}$ free induction decay signals were accumulated with the phase alternating pulse technique. In the second step the signal path was blocked for the following sequence of $2^{15}$ periods through a $1 \mathrm{GHz}$ shift of the YIG transmission band pass but data accumulation (in this case of noise and perturbations only) was carried out as before. In the third step the difference between both accumulated signals was built and added to the results of the previous three-step cycles. By this technique the remaining coherent perturbations, which probably mostly originate from the switching pulses within the hardware of the averager system, could be further reduced by at least three orders of magnitude. This considerably facilitated the present study of the low-dipole molecule norbornadiene.

In Fig. 2 we present Fourier transform power spectra of the $2(1,1)-1(0,1)$ rotational transition as examples. They were recorded at zero field and at fields close to $17 \mathrm{kGauss}$. Typical recording conditions were: sample gas pressures around 2 mTorr and sample temperatures in the range of -40 to $-50^{\circ} \mathrm{C}$. Under these conditions linewidths of typically $50 \mathrm{kHz}$ full width at half height were observed in the Fourier transform power spectra. Due to the low dipole moment of norbornadiene comparatively long polarization pulses of up to $1.5 \mu$ s duration each had to be used for polarization of the sample. With such long pulses the resonance offset with respect to the polarization frequency already leads to observable differences in the polarization efficiency for the different Zeeman satellites. Thus the observed relative signal intensities of the satellite not only depend on the dipole transition moment matrix element but also on the frequency offset with respect to the carrier frequency in the pulse [6].

For reasons discussed earlier [7 - 10], the final transition frequencies were obtained from a direct leastsquares fit to the accumulated free induction decay signals. A Levenberg-Marquardt routine [11] implemented by Grabow [12] was used for this purpose.

\section{Analysis of the Zeeman Splittings}

The energy expression, developed by Hüttner and Flygare [13], for the magnetic contribution to the rotational energy levels of an asymmetric top molecule was used to analyze the observed Zeeman splittings. It is given by

$$
\begin{aligned}
E\left(J, K_{a} K_{c}, M\right)= & -\mu_{\text {nuc }} \cdot \frac{M \cdot H}{J(J+1)} \cdot\left(g_{a a}\left\langle J_{a}^{2}\right\rangle+g_{b b}\left\langle J_{b}^{2}\right\rangle+g_{c c}\left\langle J_{c}^{2}\right\rangle\right)-\frac{1}{2 \cdot N} \cdot \xi_{\text {bulk }} \cdot H^{2} \\
& -\frac{H^{2}}{3 \cdot N} \cdot \frac{\left(3 \cdot M^{2}-J(J+1)\right)}{(2 J-1)(2 J+3) J(J+1)}\left[\alpha\left(\left\langle J_{a}^{2}\right\rangle-\left\langle J_{c}^{2}\right\rangle\right)+\beta\left(\left\langle J_{b}^{2}\right\rangle-\left\langle J_{c}^{2}\right\rangle\right)\right],
\end{aligned}
$$

where $\alpha=\left(2 \xi_{a a}-\xi_{b b}-\xi_{c c}\right)$ and $\beta=\left(2 \xi_{b b}-\xi_{c c}-\xi_{a a}\right)$ are the magnetizability anisotropies, $N$ is Avogadro's number, $\mu_{\text {nuc }}$ is the nuclear magneton, $H$ is the magnetic field, $J, K_{a} K_{c}$, and $M$, are the rotational quantum numbers, and $\left\langle J_{g}^{2}\right\rangle=\left\langle J, K_{a} K_{c}\left|J_{g}^{2}\right| J, K_{a} K_{c}\right\rangle$ is the asymmetric top expectation value of the rotational angular momentum (in $\hbar$ ) along the $g^{\text {th }}$ principal inertia axis. The $\left\langle J_{g}^{2}\right\rangle$ 's were computed as derivatives of the rotational energies with respect to the rotational constants [14]. The closed solutions [15] for the low- $J$ rotational energy levels of interest here are presented in Table 1. The rotational constants of Bauder and coworkers [2] ( $A=4273.6281(2) \mathrm{MHz}$, $B=3610.3002(2) \mathrm{MHz}$, and $C=3186.4370(2)$ $\mathrm{MHz}$ ) were used in this context. 
Table 1. Closed solutions [15] for the rigid rotor low- $J$ rotational energy levels of interest here. $K_{a}$ and $K_{c}$ denote the $K$-quantum numbers of the limiting symmetric tops. $A, B, C$ denote the rotational constants. For the numerical calculations their values were taken from [2].

\begin{tabular}{lc}
\hline$J\left(K_{a}, K_{c}\right)$ & Energy \\
\hline $0(0,0)$ & 0 \\
$1(0,1)$ & $B+C$ \\
$1(1,1)$ & $A+C$ \\
$1(1,0)$ & $A+B$ \\
$2(1,1)$ & $A+4 B+C$ \\
$2(2,1)$ & $4 A+B+C$ \\
$2(2,0)$ & $2 A+2 B+2 C+2 \sqrt{(B-C)^{2}+(A-C)(A-B)}$ \\
\hline
\end{tabular}

The measured Zeeman splittings reported in Table 2 were least-squares fitted to the transition frequency expression obtained from (1), to give the three molecular $g$-values $\left(g_{a a}, g_{b b}, g_{c c}\right)$ and the two magnetizability anisotropies $\alpha=\left(2 \xi_{a a}-\xi_{b b}-\xi_{c c}\right)$, $\beta=\left(2 \xi_{b b}-\xi_{c c}-\xi_{c c}\right)$. These are reported in Table 3 .

The bulk magnetizability, $\xi_{\text {bulk }}$, can not be obtained from rotational Zeeman effect splittings, since its contribution cancels in the energy differences which lead to the transition frequencies. Also, in principle, only the relative sign can be determined for the molecular $g$-values, since a simultaneous change of the signs of all $g$-values and of the sign of all $M$-quantum numbers would lead to identical frequency and intensity patterns in the Zeeman multiplets (c.f Eqs. III.11 and III.12 and Table III.5 in [3]). Below we present arguments that our choice of sign (all $g$-values negative) is correct.

\section{Derived Molecular Parameters}

\subsection{Molecular Electric Quadrupole Moments (No Additional Input Required)}

From their theoretical expressions, the experimental $g$-tensor elements and the magnetizabilities may be used to derive experimental values for the vibronic ground state expectation values of the components of the molecular electric quadrupole moment, see [16] and [3], Sect. II.B:

$$
\begin{aligned}
Q_{a a}= & \frac{|e|}{2} \cdot\left[\langle 0| \sum_{n}^{\text {nuclei }} Z_{n}\left(2 \cdot a_{n}^{2}-b_{n}^{2}-c_{n}^{2}\right)\right. \\
& \left.-\sum_{j}^{\text {electrons }}\left(2 \cdot a_{j}^{2}-b_{j}^{2}-c_{j}^{2}\right)|0\rangle\right]
\end{aligned}
$$

Table 2. Frequencies of measured rotational transitions in the vibrational ground state of norbornadiene. The experimental uncertainties are estimated to be below $\pm 5 \mathrm{kHz}$ depending on the signal to noise ratio. $\Delta \nu_{(H)}=\nu_{(H)}-\nu_{0}$ are the shifts of the Zeeman satellites with respect to the zero-field frequency, $\nu_{0}$. Only Zeeman satellites with sufficient $S / N$ ratio and frequency offsets above $200 \mathrm{kHz}$ were used for the least squares fit of the molecular $g$-values and magnetizability anisotropies. Satellites not used in the least squares fit are marked by an asterisk. The uncertainties in the $H$-values are estimated to be below $\pm 20 \mathrm{G}$ (see [3],

\begin{tabular}{|c|c|c|c|c|c|c|c|}
\hline $\begin{array}{l}J^{\prime}\left(K_{a}^{\prime}, K_{c}^{\prime}\right) \\
-J\left(K_{a}, K_{c}\right)\end{array}$ & $M^{\prime}$ & $M$ & $\begin{array}{c}\nu_{0} \\
/ \mathrm{MHz}\end{array}$ & $\begin{array}{l}\Delta \nu_{(H)}^{\exp } \\
/ \mathrm{kHz}\end{array}$ & $\begin{array}{c}\Delta \nu_{(H)}^{\text {calc }} \\
/ \mathrm{kHz}\end{array}$ & $\begin{array}{c}I_{\text {rel }} \\
\%\end{array}$ & $\begin{array}{c}H \\
\text { /Gauss }\end{array}$ \\
\hline \multirow[b]{2}{*}{$1(0,1)-0(0,0)$} & -1 & 0 & \multirow[b]{2}{*}{7883.925} & -774 & -779.3 & 50 & \multirow[b]{2}{*}{20556} \\
\hline & & & & & & $50-5$ & \\
\hline $1(1,0) \quad 0(0 \Omega)$ & 0 & 0 & 7882025 & $260 *$ & 2606 & 100 & 10745 \\
\hline \multirow{4}{*}{$2(1,1)-1(0,1)$} & -1 & 0 & & $\begin{array}{r}-455 \\
-838\end{array}$ & -454.2 & 15 & \multirow{4}{*}{17101} \\
\hline & +2 & +1 & 15104.519 & +555 & +552.7 & 30 & \\
\hline & +1 & 0 & & +591 & +592.4 & 15 & \\
\hline & 0 & -1 & & $+490^{*}$ & +495.9 & 5 & \\
\hline \multirow{3}{*}{$2(1,1)-1(0,1)$} & +1 & +1 & & +242 & +242.0 & 30 & \multirow{3}{*}{17074} \\
\hline & 0 & 0 & 15104.519 & $+137^{*}$ & +139.7 & 40 & \\
\hline & -1 & -1 & & $-97^{*}$ & -98.4 & 30 & \\
\hline \multirow{5}{*}{$2(2,0)-1(1,0)$} & -1 & 0 & & -605 & -606.3 & 15 & \multirow{5}{*}{17101} \\
\hline & -2 & -1 & & -313 & -313.9 & 30 & \\
\hline & +2 & +1 & 16155.026 & +385 & +387.2 & 30 & \\
\hline & +1 & 0 & & +276 & +274.3 & 15 & \\
\hline & 0 & -1 & & $+693^{*}$ & +690.3 & 5 & \\
\hline \multirow{3}{*}{$2(2,0)-1(1,0)$} & +1 & +1 & & $+35^{*}$ & +39.4 & 30 & \multirow{3}{*}{17074} \\
\hline & 0 & 0 & 16155.026 & $-132^{*}$ & -134.7 & 40 & \\
\hline & -1 & -1 & & +215 & +218.5 & 30 & \\
\hline \multirow{6}{*}{$2(2,1)-1(1,1)$} & 0 & +1 & & $-299 *$ & -296.6 & 5 & \multirow{6}{*}{17101} \\
\hline & -1 & 0 & & $-164^{*}$ & -167.9 & 15 & \\
\hline & -2 & -1 & 16431.171 & -637 & -634.7 & 30 & \\
\hline & +2 & +1 & & +438 & +438.6 & 30 & \\
\hline & +1 & 0 & & +565 & +564.4 & 15 & \\
\hline & 0 & -1 & & $+92^{*}$ & +94.8 & 5 & \\
\hline \multirow{3}{*}{$2(2,1)-1(1,1)$} & +1 & +1 & & $+75^{*}$ & +70.3 & 30 & \multirow{3}{*}{17074} \\
\hline & 0 & 0 & 16431.171 & $+197^{*}$ & +196.9 & 40 & \\
\hline & -1 & -1 & & -268 & -270.1 & 30 & \\
\hline
\end{tabular}
p. 118).

$$
\begin{aligned}
Q_{a a}= & -\frac{\hbar|e|}{8 \pi M_{p}} \cdot\left(\frac{2 g_{a a}}{A}-\frac{g_{b b}}{B}-\frac{g_{c c}}{C}\right) \\
& -\frac{2 m c^{2}}{|e| N} \cdot\left(2 \xi_{a a}-\xi_{b b}-\xi_{c c}\right)
\end{aligned}
$$

where $A, B$, and $C$ are the rotational constants, $M_{p}$ and $m$ are the masses of the proton and electron, respectively, $Z_{n}|e|$ is the charge of the $n$-th nucleus, $a_{n}, b_{n}, c_{n}, a_{j}, b_{j}, c_{j}$ are the coordinates of the $n$-th 
Table 3. Molecular Zeeman parameters for norbornadiene, which follow directly from the observed Zeeman splittings and the rotational constants. A positive sign for the $g$-values would lead to unrealistic values for the molecular electric quadrupole moments, given at the bottom of the Table. The uncertainties correspond to single standard deviations from the least squares fit to the observed Zeeman splittings.

\begin{tabular}{|c|c|c|c|}
\hline Molecular $g$-values & $\begin{array}{l}g_{a a} \\
g_{b b} \\
g_{c c}\end{array}$ & $\begin{array}{l}-0.02860 \pm \\
-0.05271 \pm \\
-0.00142 \pm\end{array}$ & $\begin{array}{l}0.00016 \\
0.00011 \\
0.00026\end{array}$ \\
\hline $\begin{array}{l}\text { Magnetizability } \\
\text { anisotropies }\end{array}$ & $2 \xi_{a a}-\xi_{b b}-\xi_{c c}$ & $-0.41 \pm$ & 0.26 \\
\hline$/ 10^{-6} \mathrm{ergG}^{-2} \mathrm{~mol}^{-1}$ & $2 \xi_{b b}-\xi_{c c}-\xi_{a a}$ & $40.72 \pm$ & 0.27 \\
\hline $\begin{array}{l}\text { Molecular quadrupole } \\
\text { moments }\end{array}$ & $Q_{a a}$ & $-1.784 \pm$ & 0.20 \\
\hline (all $g$-values negative) & $Q_{b b}$ & $5 \pm$ & 0.20 \\
\hline$/ 10^{-26}$ esu cm ( D A $)$ & $Q_{c c}$ & $-1.942 \pm$ & 0.30 \\
\hline $\begin{array}{l}\text { Molecular quadrupole } \\
\text { moments }\end{array}$ & $Q_{a a}$ & $2.248 \pm$ & 0.20 \\
\hline (all $g$-values positive) & $Q_{b b}$ & $-49.83 \pm$ & 0.20 \\
\hline$/ 10^{-26}$ esu cm ( D $\AA$ ) & $Q_{c c}$ & $47.56 \pm$ & 0.30 \\
\hline
\end{tabular}

nucleus and $j$-th electron, respectively, in the principal moment of inertia coordinate system, $c$ is the velocity of light, and $\langle 0|\ldots| 0\rangle$ denotes the vibronic ground state expectation value.

Depending on the choice of sign for the $g$-values, two completely different sets of molecular electric quadrupole moments are obtained from (3). However, the absolute values of the quadrupole moments which would follow from the set with positive $g$-values have the order of magnitude of the nuclear contributions alone (see Table 3). Since the nuclear and electronic contributions largely compensate each other, these values would be too large by an order of magnitude. This conclusively demonstrates that our choice of sign for the $g$-values (all negative) must be correct.

With additional input, also the so-called paramagnetic magnetizabilities, the anisotropies in the second electronic moments, the individual components of the magnetizability tensor and the individual second electronic moments may be obtained.

\subsection{Paramagnetic Magnetizabilities and Anisotropies} in the Second Moments of the Electronic Charge Distribution. (Additional Input: Second Moments of the Nuclear Charge Distribution. These are derived from the microwave structure [2])

With the second moments of the nuclear charge distribution, $\sum Z_{n} a_{n}^{2}, \sum Z_{n} b_{n}^{2}$, and $\sum Z_{n} c_{n}^{2}$ as additional input, also the components of the paramagnetic magnetizability and the anisotropies in the second moments of the electronic charge distribution were calculated with the following equations ([3], p. 98):

\section{Paramagnetic magnetizabilities:}

$$
\begin{aligned}
& \xi_{a a}^{(p)}=-\frac{N e^{2}}{2 m^{2} c^{2}} \cdot\left(\frac{L_{a} L_{a}}{\Delta}\right), \\
& \xi_{a a}^{(p)}=\left(-\frac{e^{2} N}{4 m c^{2}}\right) \cdot\left[\frac{\hbar}{4 \pi M_{p}} \cdot \frac{g_{a a}}{A}\right. \\
&\left.-\left\langle 0\left|\sum_{n}^{\text {nuclei }} Z_{n}\left(b_{n}^{2}+c_{n}^{2}\right)\right| 0\right\rangle\right]
\end{aligned}
$$

(and cyclic permutations). In (4), $\left(L_{a} L_{a} / \Delta\right)$ is a second-order perturbation sum running over the excited electronic states of the molecule (see Chapts. I and IV in [3]).

Anisotropies in the second moments of the electronic charge distribution:

$$
\begin{aligned}
& \left\langle 0\left|\sum_{j}^{\text {electrons }}\left(a_{j}^{2}-b_{j}^{2}\right)\right| 0\right\rangle=\left\langle 0\left|\sum_{n}^{\text {nuclei }} Z_{n}\left(a_{n}^{2}-b_{n}^{2}\right)\right| 0\right\rangle \\
& \quad+\frac{\hbar}{4 \pi M_{p}}\left(\frac{g_{a a}}{A}-\frac{g_{b b}}{B}\right)+\frac{4 m c^{2}}{3 \cdot N \cdot e^{2}} \\
& \quad \cdot\left[\left(2 \xi_{a a}-\xi_{b b}-\xi_{c c}\right)-\left(2 \xi_{b b}-\xi_{c c}-\xi_{a a}\right)\right]
\end{aligned}
$$

The values of $\sum Z_{n} a_{n}^{2}, \sum Z_{n} b_{n}^{2}$, and $\sum Z_{n} c_{n}^{2}$ needed in these equations were derived from the microwave structures. Three sets of coordinates were used: the $r_{0}$-coordinates, the $r_{\mathrm{s}}$-coordinates, and the pseudo- $r_{\mathrm{e}}$-coordinates. The substitution $\left(r_{\mathrm{s}}\right)$ coordinates and the pseudo-equilibrium (pseudo- $r_{\mathrm{e}}$ ) coordinates (compare [17]) were taken from Table II in [2]. The $r_{0}$-coordinates are the result of a least-squares fit to the vibronic ground state rotational constants of all isotopomers reported in [2]. They are presented in Table 4 . The rigid nuclear frame approximation was used in this fit with the same nuclear frame geometry for all isotopomers. For later reference we also present Hartree-Fock self consistent field equilibrium coordinates in this Table. They were calculated using the aug-cc-pVDZ basis of Dunning and coworkers (see Sect. 5.1). 
Table 4. Nuclear coordinates $(\AA)$ of norbornadiene in the principal inertia axes system. For the numbering of the carbon atoms compare Figure 1.

\begin{tabular}{cccrrrrr}
\hline Nucleus & \multicolumn{3}{c}{$r_{0}$-coordinates } & \multicolumn{3}{c}{ SCF- $r_{\mathrm{e}}$-coordinates } \\
& $a$ & $b$ & $c$ & $a$ & $b$ & \multicolumn{1}{c}{$c$} \\
\hline $1-\mathrm{C}$ & 0.0000 & 1.1207 & 0.2926 & 0.0000 & 1.1165 & 0.2852 \\
$2-\mathrm{C}$ & 1.2382 & 0.6734 & -0.5118 & 1.2404 & 0.6624 & -0.5087 \\
$7-\mathrm{C}$ & 0.0000 & 0.0000 & 1.3656 & 0.0000 & 0.0000 & 1.3641 \\
$1-\mathrm{H}$ & 0.0000 & 2.1557 & 0.6259 & 0.0000 & 2.1492 & 0.6221 \\
$2-\mathrm{H}$ & 1.9228 & 1.3329 & -1.0142 & & 1.9343 & 1.3237 & -1.0038 \\
$7-\mathrm{H}$ & 0.9090 & 0.0000 & 1.9764 & 0.8950 & 0.0000 & 1.9837 \\
\hline
\end{tabular}

Table 5. Molecular Zeeman parameters calculated with the second moments of the nuclear charge distribution as additional input. Four sets of data are presented. They correspond to the microwave $r_{0^{-}}, r_{\mathrm{s}}-$, pseudo- $r_{\mathrm{e}}$-structures and to a fully relaxed SCF-structure, respectively (see text). The uncertainties follow by Gaussian error propagation from the experimental uncertainties in the $g$-values and magnetizability anisotropies alone. They correspond to single standard deviations and are given in units of the least significant figure. The differences between the four columns provide an impression of the size of vibrational corrections.

\begin{tabular}{lrrrr}
\hline & \multicolumn{1}{c}{$r_{0}$} & \multicolumn{1}{c}{$r_{\mathrm{S}}$} & pseudo- $r_{\mathrm{e}}$ & $r_{\mathrm{e}(\mathrm{SCF})}$ \\
\hline$\sum Z_{n} \cdot a_{n}^{2} / \AA^{2}$ & 53.2380 & 53.1366 & 53.0099 & 53.492 \\
$\Sigma Z_{n} \cdot b_{n}^{2} / \AA^{2}$ & 42.3543 & 42.2453 & 42.1045 & 41.738 \\
$\Sigma Z_{n} \cdot c_{n}^{2} / \AA^{2}$ & 31.2134 & 31.0544 & 30.8268 & 31.025 \\
$\xi_{a a}^{(p)} /\left(10^{-6} \mathrm{erg} \mathrm{G}^{-2} \mathrm{~mol}^{-1}\right)$ & $326.46(08)$ & $325.32(08)$ & $323.76(08)$ & $323.05(08)$ \\
$\xi_{b b}^{(p)} /\left(10^{-6} \mathrm{erg} \mathrm{G}^{-2} \mathrm{~mol}^{-1}\right)$ & $389.59(07)$ & $388.48(07)$ & $386.98(08)$ & $389.87(08)$ \\
$\xi_{c c}^{(p)} /\left(10^{-6} \mathrm{erg} \mathrm{G}^{-2} \mathrm{~mol}^{-1}\right)$ & $406.51(18)$ & $405.61(18)$ & $404.48(18)$ & $404.97(18)$ \\
$\left\langle\left|\Sigma\left(a_{j}^{2}-b_{j}^{2}\right)\right|\right\rangle / \AA^{2}$ & $11.65(4)$ & $11.66(4)$ & $11.67(4)$ & $12.52(4)$ \\
$\left\langle\left|\Sigma\left(b_{j}^{2}-c_{j}^{2}\right)\right|\right\rangle / \AA^{2}$ & $10.35(7)$ & $10.40(7)$ & $10.49(7)$ & $9.93(7)$ \\
$\left\langle\left|\Sigma\left(c_{j}^{2}-a_{j}^{2}\right)\right|\right\rangle / \AA^{2}$ & $-22.00(7)$ & $-22.06(7)$ & $-22.16(7)$ & -22.44 \\
\hline
\end{tabular}

The input values for the second nuclear moments, i. e. for the sums $\sum Z_{n} a_{n}^{2}$ etc., are given at the top of Table 5. Also given in this Table are the corresponding values for a fully relaxed ab-initio geometry (SCF-calculation, aug-cc-pVDZ basis; see the following section). The corresponding paramagnetic magnetizabilities and anisotropies in the second moments of the electronic coordinates are presented at the bottom of Table 5. The uncertainties given in this Table follow by Gaussian error propagation from the experimental uncertainties in the $g$-values and magnetizability anisotropies alone. They do not account for model deficiencies due to the neglect of vibrational corrections. We believe however, that the differences between the values in the four columns provide an estimate for the order of magnitude of vibrational corrections.
Table 6. Prediction of the bulk magnetizability of norbornadiene (in units of $10^{-6} \mathrm{ergG}^{-2} \mathrm{~mol}^{-1}$ ) using Haberditzl's additivity scheme for core and bond contributions (see p. $96-97$ of [18]).

\begin{tabular}{llr}
\hline 8 H cores & $(0.0$ each $)$ & 0.00 \\
7 C cores & $(-0.15$ each $)$ & -1.05 \\
$2 \mathrm{C}_{2}^{+} \pi \mathrm{C}_{2}^{+}$ & $(-2.2$ each $)$ & -4.4 \\
$2 \mathrm{C}^{+}-\mathrm{C}^{+}$ & $(-2.6$ each $)$ & -5.2 \\
$4 \mathrm{C}-\mathrm{C}^{+}$ & $(-2.6$ each $)$ & -10.4 \\
$2 \mathrm{C}_{2}-\mathrm{C}_{3}$ & $(-3.4$ each $)$ & -6.8 \\
$4 \mathrm{C}_{2}^{+}-\mathrm{H}$ & $(-3.2$ each $)$ & -12.8 \\
$2 \mathrm{C}_{3}-\mathrm{H}$ & $(-3.5$ each $)$ & -7.0 \\
$2 \mathrm{C}_{2}-\mathrm{H}$ & $(-3.8$ each $)$ & -7.6 \\
$\xi_{\text {bulk }}^{\text {(pred) }}-$ norbornadiene & & -55.25 \\
\hline
\end{tabular}

4.3. The Individual Components of the Magnetizability Tensor and the Individual Second Moments of the Electronic Charge Distribution. (Additional Input: the Second Moments of the Nuclear Charge Distribution (as above) and the Bulk Magnetizability, $\left.\xi_{\text {bulk }}=\left(\xi_{a a}+\xi_{b b}+\xi_{c c}\right) / 3\right)$

Since no experimental value for the bulk magnetizability was known to us, we have used Haberditzl's additivity scheme for core and bond increments [18] to predict its value. For unstrained hydrocarbons, this additivity scheme typically leads to predictions within $1 \%$ of the experimental (liquid phase) values [18]. In Table 6 we present an excerpt of Haberditzl's Tables.

In Table 6 a plain " $\mathrm{C}$ " stands for a $\mathrm{sp}^{3}$-hybridized carbon atom. A $\mathrm{sp}^{2}$-hybridized carbon atom is denoted by " $\mathrm{C}^{+}$". Lower indices $n=1,2,3$, denote the number of neighbouring carbon atoms, which are attached to the atom under consideration (compare with Figure 1). The result of such a prediction is

$$
\xi_{\text {bulk }}^{\text {(pred) }}=-55.25 \cdot 10^{-6} \mathrm{ergG}^{-2} \mathrm{~mol}^{-1} .
$$

Because of the presence of strain, the uncertainty in this value will be larger than the typical $1 \%$ margin observed for unstrained hydrocarbons [18]. Also, a difference in the gas-phase and liquid-phase magnetizability tensors will increase the uncertainties in the derived molecular parameters. In fact, there are indications that the gas-to-liquid shift of the magnetizability may be as large as 10 to $15 \%$, with the value in liquid being more paramagnetic than in the gas phase [19]. In Table 7, we therefore also present values calculated with a bulk magnetizability $10 \%$ 
Table 7. Molecular Zeeman parameters $(I)$ calculated with the second moments of the nuclear charge distribution and a predicted value for the bulk magnetizability as additional input. Two sets of data are presented for each nuclear configuration. The second set $(I I)$ is calculated with a $\xi_{\text {bulk }}$-value increased by $10 \%$ with respect to the Haberditzl-prediction (see text). The uncertainties reflect the experimental uncertainties in the $g$-values and magnetizability anisotropies alone. The magnetizabilities are given in units of $10^{-6} \mathrm{ergG}^{-2} \mathrm{~mol}^{-1}$; the second moments in units of $\AA^{2}$.

\begin{tabular}{|c|c|c|c|c|c|c|c|c|}
\hline & $r_{0}^{(\mathrm{I})}$ & $r_{0}^{(\mathrm{II})}$ & $r_{\mathrm{s}}^{(\mathrm{I})}$ & $r_{\mathrm{s}}^{(\mathrm{III})}$ & pseudo- $r_{\mathrm{e}}^{(\mathrm{I})}$ & pseudo- $r_{\mathrm{e}}^{(\mathrm{II})}$ & $r_{\mathrm{e}(\mathrm{SCF})}^{(\mathrm{I})}$ & $r_{\mathrm{e}(\mathrm{SCF})}^{(\mathrm{III})}$ \\
\hline $\begin{array}{l}\xi_{\text {bulk }} \\
\xi_{a a} \\
\xi_{b b} \\
\xi_{c c}\end{array}$ & $\begin{array}{l}-55.25 \\
-55.39(9) \\
-41.68(9) \\
-68.69(12)\end{array}$ & $\begin{array}{l}-60.77 \\
-60.91 \\
-47.20 \\
-74.21\end{array}$ & $\begin{array}{l}-55.25 \\
-55.39 \\
-41.68 \\
-68.69\end{array}$ & $\begin{array}{l}-60.77 \\
-60.91 \\
-47.20 \\
-74.21\end{array}$ & $\begin{array}{l}-55.25 \\
-55.39 \\
-41.68 \\
-68.69\end{array}$ & $\begin{array}{l}-60.77 \\
-60.91 \\
-47.20 \\
-74.21\end{array}$ & $\begin{array}{l}-55.25 \\
-55.39 \\
-41.68 \\
-68.69\end{array}$ & $\begin{array}{l}-60.77 \\
-60.91 \\
-47.20 \\
-74.21\end{array}$ \\
\hline$\left\langle 0\left|\Sigma a_{j}^{2}\right| 0\right\rangle$ & $61.83(3)$ & 62.48 & 61.73 & 62.38 & 61.60 & 62.25 & 62.08 & 62.73 \\
\hline$\left\langle 0\left|\Sigma b_{j}^{2}\right| 0\right\rangle$ & $50.18(3)$ & 50.83 & 50.07 & 50.72 & 49.93 & 50.58 & 49.56 & 50.21 \\
\hline$\left\langle 0\left|\Sigma c_{j}^{2}\right| 0\right\rangle$ & $39.83(4)$ & 40.48 & 39.67 & 40.32 & 39.44 & 40.09 & 39.64 & 40.29 \\
\hline
\end{tabular}

more diamagnetic than the Haberditzl prediction as additional input.

With $\xi_{\text {bulk }}^{\text {(pred.) }}$ and with the experimental magnetizability anisotropies from Table 3, the individual components of the magnetizability tensor may be calculated, and the individual second moments of the electronic charge distribution then follow as given in (7):

$$
\begin{aligned}
& \left\langle 0\left|\sum_{j}^{\text {electrons }} b_{j}^{2}\right| 0\right\rangle=\frac{2 m c^{2}}{e^{2} N} \cdot\left(\xi_{b b}-\xi_{c c}-\xi_{a a}\right) \\
& +\frac{\hbar}{8 \pi M_{p}} \cdot\left(\frac{g_{b b}}{B}-\frac{g_{c c}}{C}-\frac{g_{a a}}{A}\right)+\left\langle 0\left|\sum_{n}^{\text {nuclei }} Z_{n} b_{n}^{2}\right| 0\right\rangle
\end{aligned}
$$

(and cyclic permutations). These quantities are listed at the bottom of Table 7. As already mentioned, because of the strain-which leads to an endo displacement of the hydrogen atoms attached to the $\mathrm{sp}^{2}$ hybridized carbon atoms, for instance-and because the increments of the additivity scheme used here have been fitted to predicted liquid-phase bulk magnetizabilities, these results have to be regarded with some caution.

In an alternative approach we have therefore calculated the magnetic properties of norbornadiene directly by a powerful ab-initio response method using London type atomic orbitals as basis functions. This is described in the following section.

\section{Ab initio Calculations}

\subsection{Introductory Remarks}

For a critical comparison with the experimental values, we have calculated - at the Hartree-Fock levelthe magnetizability and rotational $g$ tensors as well as the dipole and quadrupole moments of the norbornadiene molecule. For the magnetic properties, our use of London atomic orbitals ensures gauge-origin independence and fast basis-set convergence [20, 21]. In our calculations, we have used the augmented correlation-consistent polarized valence double-zeta basis (aug-cc-pVDZ) of Dunning and coworkers, developed to give accurate results for electric properties $[22,23]$. It has been shown that this basis set gives Hartree-Fock results within $2 \%$ of the Hartree-Fock limit for magnetizabilities as well as rotational $g$ tensors $[21,24,25]$. The aug-cc-pVDZ set consists of a $(4 \mathrm{~s} 3 \mathrm{p} 2 \mathrm{~d})$ contracted set on carbon and a $(3 \mathrm{~s} 2 \mathrm{p})$ contracted set on hydrogen, giving a total of 233 basis functions for this molecule.

In our calculations, we have used the substitution coordinate geometry and the pseudo-equilibrium geometry of the Zürich group [2]. In addition, we have performed a geometry optimization at the aug-ccpVDZ level in order to obtain a purely theoretical estimate of the various molecular parameters. The geometry was optimized using the second-order algorithm described in [26]. All calculations have been performed with the DALTON quantum chemistry program [27].

The use of London atomic orbitals ensures that the results obtained with the aug-cc-pVDZ basis set employed in this investigation are within $2 \%$ of the Hartree-Fock limit [20, 24]. Furthermore, the inclusion of electron-correlation effects usually leads to minor changes in the isotropic magnetizability (of the order of $2 \%$ ) [28] - often in the opposite direction of the error caused by basis-set incompleteness. Thus, we estimate the $\xi_{\text {bulk-values obtained with the }}$ London atomic orbitals to be accurate to within $3 \%$. 
Table 8. Molecular Zeeman parameters as obtained from $a b$-initio calculations. The first and second columns refer to the experimental pseudo- $r_{\mathrm{e}}$ and $r_{\mathrm{s}}$-geometries, respectively, taken from [2]. The last column refers to the fully relaxed SCF-geometry (see text). The units are: $10^{-6} \mathrm{ergG}^{-2} \mathrm{~mol}^{-1}$ for the magnetzabilities, esu $\AA$ (D) for the dipole moment, and $10^{-26}$ esu $\mathrm{cm}^{2}$ (D $\AA$ ) for the molecular quadrupole moment.

\begin{tabular}{lrrr}
\hline & pseudo- $r_{\mathrm{e}}$ & \multicolumn{1}{c}{$r_{\mathrm{s}}$} & aug-cc-pVDZ \\
\hline & & & \\
$\xi_{a a}$ & -60.07 & -60.31 & -60.44 \\
$\xi_{b b}$ & -47.81 & -47.86 & -48.12 \\
$\xi_{c c}$ & -73.20 & -73.23 & -73.69 \\
$\xi \xi_{a a}-\xi_{b b}-\xi_{c c}$ & -60.36 & -60.47 & -60.75 \\
$2 \xi_{b b}-\xi_{c c}-\xi_{a a}$ & 0.87 & 0.47 & 0.93 \\
$\xi_{a a}^{(p)}$ a & 37.65 & 37.82 & 37.89 \\
$\xi_{b b}^{(p)}$ & 322.6 & 323.9 & 320.5 \\
$\xi_{c c}^{(p)}$ & 385.3 & 386.7 & 383.4 \\
$g_{a a}$ & 403.4 & 404.5 & 397.6 \\
$g_{b b}$ & & & \\
$g_{c c}$ & -0.0267 & -0.0261 & -0.0240 \\
$\mu$ & -0.0506 & -0.0503 & -0.0421 \\
$\mu$ & 0.0001 & 0.0002 & +0.0096 \\
$Q_{a a}$ & & & \\
$Q_{b b}$ & 0.0166 & 0.0299 & 0.0640 \\
$Q_{c c}$ & & & \\
\hline & -2.29 & -2.31 & -2.08 \\
\hline & 4.76 & 4.81 & 4.58 \\
& -2.48 & -2.51 & -2.50 \\
\hline
\end{tabular}

a The paramagnetic term is defined as $\xi_{\text {lon }}-\xi_{\text {nolon }}^{\text {dia }}$, where $\xi_{\text {nolon }, a a}^{\text {dia }}=-\frac{e^{2}}{4 m c^{2}}\left\langle 0\left|\Sigma\left(b_{j}^{2}+c_{j}^{2}\right)\right| 0\right\rangle$ etc.

At the substituted coordinate structure of the Zürich group [2], the isotropic magnetizability becomes

$$
\xi_{\mathrm{SCF}}^{\text {pred }}=-60.4 \pm 1.8 \cdot 10^{-6} \mathrm{erg} \mathrm{G}^{-2} \mathrm{~mol}^{-1},
$$

a value which is about $9 \%$ more diamagnetic than that predicted by Haberditzl's additivity scheme (compare Table 6).

\subsection{Ab-initio Calculations of the Electric and Mag- netic Properties of Norbornadiene}

We have collected our calculated results for the dipole moment, the quadrupole moment, the rotational $g$ tensor, the magnetizability tensor, and its paramagnetic part in Table 8 . We note that the various molecular properties depend only weakly on the geometry. We thus expect that rovibrational effects will have only a minor influence on the calculated properties-with the possible exceptions of the magnetizability anisotropy $2 \xi_{a a}-\xi_{b b}-\xi_{c c}$ and the isotropic magnetizability.
Comparing the Hartree-Fock results in Table 8 with those obtained directly from experiment-that is, with the rotational $g$ tensor elements and the magnetizability anisotropies in Table 3-we note that the agreement is satisfactory for the $g$ tensor components $g_{a a}$ and $g_{b b}$, the difference being approximately 6.5 and $4.0 \%$, respectively. This appears to be the accuracy characteristic of Hartree-Fock calculations for this particular property $[21,28]$. For the $g_{c c}$ component, however, the theoretical results have, at all geometries, a sign opposite that assigned by experiment. Considering its small magnitude, the inclusion of electron correlation might change the sign of this component. The calculations thus give a straightforward and unambiguous verification of the sign of the rotational $g$ tensor components chosen on the basis of the predicted quadrupole moment.

The isotropic magnetizability as obtained in the theoretical calculations confirm the isotropic magnetizability obtained using Haberditzl's additivity scheme after the scaling of $10 \%$. For the magnetizability anisotropy $2 \xi_{a a}-\xi_{b b}-\xi_{c c}$, the calculated results are of the same order of magnitude as experiment but of opposite sign. Comparing with Table 7 , we note that this difference appears to arise from a slight overestimation of the $\xi_{b b}$ component in the theoretical calculation, which is also the cause of the fairly large difference observed for the second magnetizability anisotropy. The neglect of electron correlation usually introduces errors in the magnetizability components that tend to cancel for the isotropic magnetizability but which lead to poorer agreement with experiment for the anisotropies [29]. We note that, for the paramagnetic contributions to the magnetizability, there is good agreement with the experimentally derived numbers in Table 5.

Turning our attention to the quadrupole moment, the agreement between theory and experiment is satisfactory, although none of the theoretical estimates are within the experimental error bars. However, the quadrupole moments result from the differences of large nuclear and large electronic contributions. This leads to their comparatively high sensitivity with respect to even minor errors in the calculated electronic wavefunction.

\subsection{Ring Currents}

Considering the geometry of norbornadiene as depicted in Figure 1, we note the possibility of overlap 
of the $p$ orbitals on the doubly-bonded carbon atoms, which in turn might lead to ring currents. The importance of ring currents for the magnetizability of aromatic molecules was studied already in the 1930 s [30] and has recently been investigated in detail by Schleyer and Jiao [31]. The existence of a closed path where electrons may move freely will lead to an enhancement of the magnetizability, for both the isotropic and the anisotropic magnetizability. Thus, large magnetizability anisotropies and magnetizability exaltations - as defined with respect to an additivity scheme-may indicate the existence of ring currents and thus serve as a measure of the aromaticity of a molecule [31].

To estimate possible magnetizability exaltations, we first predicted the tensor elements $\xi_{g g}$ from local atom tensors as outlined in Chapt. $\mathrm{C}$ of [3]. The local tensors were taken from Table II.2 in [3]. Unfortunately, as a result of the large uncertainties in these local tensor elements (typically in the $10-15$ $\%$ range), which probably reflect adjacent bond effects, the $\xi$-values reported in column 1 of Table 7 fall within the error bars of the predicted values, making the comparison inconclusive. Furthermore, since liquid-phase bulk magnetizabilities were used as input data for Table II.2 in [3], we now believe that the local tensor elements reported in [3] also contain a systematic error, being too paramagnetic.

Our second approach, therefore, is based on the comparison of our calculated isotropic Hartree-Fock magnetizability at the optimized geometry, $\xi_{\text {bulk }}^{\mathrm{SCF}}=$ $-60.75 \cdot 10^{-6} \mathrm{ergG}^{-2} \mathrm{~mol}^{-1}$, with the value of -59.0 . $10^{-6} \mathrm{ergG}^{-2} \mathrm{~mol}^{-1}$, obtained by adding the theoretically calculated atom magnetizabilities from [24]. The theoretical additivity parameters in [24] have been shown to give results in excellent agreement with gas phase measurements after corrections were made for systematic errors in the experimental numbers [24]. Thus, judged on the basis of the theoretical calculations alone, there appears to be only a modest magnetizability exaltation, amounting to approximately $-1.7 \cdot 10^{-6} \mathrm{ergG}^{-2} \mathrm{~mol}^{-1}$ in the bulk value or to at most $\Delta \xi_{c c}^{\text {(exalt) }}=-5.1 \cdot 10^{-6} \mathrm{ergG}^{-2} \mathrm{~mol}^{-1}$ if confined to the $\xi_{c c}$-value alone.

Although this result might indicate that a weak ring current may be induced in the $\pi$-orbital system, this current is by no means comparable to the currents observed in aromatic $\pi$-systems such as in benzene, thiophene, and so on, where the $\xi$-tensor element perpendicular to the aromatic ring may show magnetizability exaltations of more than $-40 \cdot 10^{-6} \mathrm{ergG}^{-2} \mathrm{~mol}^{-1}$ (c. f. Table II.4 in [3]).

\section{Conclusions}

From our comparison of the experimental $g$ values and $\xi$ anisotropies with their SCF counterparts, we draw three conclusions:

1. The SCF calculations predict the $g$ tensor elements with an uncertainty of about \pm 0.002 and the $\xi$ tensor elements with an uncertainty of $\pm 1.5 \cdot 10^{-6} \mathrm{ergG}^{-2} \mathrm{~mol}^{-1}$.

2. The gas-phase isotropic magnetizability $\left(\xi_{a a}+\right.$ $\left.\xi_{b b}+\xi_{c c}\right) / 3$ is most likely about $8.5 \%$ more diamagnetic than predicted from Haberditzl's additivity scheme for liquid-phase isotropic magnetizabilities, quite in line with earlier findings for compounds where both gas-phase and liquid-phase isotropic magnetizabilities have been determined experimentally.

3. Diamagnetic magnetizability exaltation due to strain-induced $\pi$ overlap-if at all present-is much less pronounced than anticipated.

In order to investigate further the effects of strain on the magnetic properties of small molecules, a joint experimental/theoretical investigation of substituted cyclo-propanes is under way.

\section{Acknowledgements}

K. V. and D. H. S would like to thank V. Storm, $\mathrm{K}$. Gerke and all other members of the Kiel group for helpful discussions and Prof. Dr. H. Dreizler for critically reading the manuscript. The assistance of Dr. R. Schwarz and H. Feldmeier from the electronics team and of the members of our machine shop was of invaluable help. Funding by Deutsche Forschungsgemeinschaft under Grant Su 41/17-1 and also by Fonds der Chemie is gratefully acknowledged. This work has received support from the Norwegian Supercomputer Committee (TRU) through a grant of computer time. 
[1] B. Vogelsanger and A. Bauder, J. Mol. Spectrosc. 130, 249 (1988).

[2] G. Knuchel, G. Grassi, B. Vogelsanger, and A. Bauder, J. Amer. Chem. Soc. 115, 10845 (1993).

[3] D. H. Sutter and W. H. Flygare, The Molecular Zeeman Effect, Top. Curr. Chem. Vol 63, 91 (1976).

[4] H. Dreizler, Ber. Bunsenges. Phys. Chem. 99, 1451 (1995).

[5] O. Böttcher, B. Kleibömer, and D. H. Sutter, Ber. Bunsenges. Phys. Chem. 93, 207 (1989) .

[6] J. C. McGurck, T. G. Schmalz, and W. H. Flygare, Adv. Chem. Phys. 25, 1 (1974), eqs. (134) and (147).

[7] H. Barkhuisen, R. de Beer, W. M. Bovee, and D. van Ormondt, J. Magn. Res. 61, 465 (1985).

[8] O. Böttcher and D. H. Sutter, Z. Naturforsch. 43a, 47 (1989).

[9] I. Merke and H. Dreizler, Z. Naturforsch. 43a, 196 (1989).

[10] J. Haeckel and H. Mäder, Z. Naturforsch. 43a, 203 (1989).

[11] W. H. Press, S. A. Teukolsky, W. T. Vetterling, and B. P. Flannery, Numerical Recipies in Fortran, $2^{\text {nd }}$ Ed., Univ. Press, Cambridge 1992, p. 678.

[12] J. U. Grabow, PhD-thesis, Christian-Albrechts-Universität Kiel (1994).

[13] W. Hüttner and W. H. Flygare, J. Chem. Phys. 47, 4137 (1967).

[14] L. Landau and E. M. Lifshitz, Lehrbuch der Theorettischen Physik III, Chapt. II, Sect. 11, Exercise.

[15] Walter Gordy and Robert L. Cook, Microwave Molecular Spectra $3^{\text {rd }}$ Ed., Table 7-7, John Wiley, New York 1982.

[16] W. Hüttner, M. K. Lo, and W. H. Flygare, J. Chem. Phys. 48, 1206 (1968).

[17] M. D. Harmony and W. H. Taylor, J. Mol. Spectrosc. 118, 163 (1986).
[18] Werner Haberditzl, Über ein neues DiamagnetismusInkrementensystem, Sitzungsberichte der Deutschen Akademie der Wissenschaften, Klasse für Chemie, Geologie und Biologie, Berlin, Jahrg. 1964, No. 2.

[19] K. V. Mikkelsen, K. Ruud, and T. Helgaker, Chem. Phys. Lett., 253, 443 (1996).

[20] K. Ruud, T. Helgaker, K. L. Bak, P. Jørgensen, and H. J. Aa. Jensen, J. Chem. Phys., 99, 3847 (1993).

[21] J. Gauss, K. Ruud, and T. Helgaker, J. Chem. Phys., 105, 2804 (1996).

[22] T. H. Dunning Jr. J. Chem. Phys., 90, 1007 (1989).

[23] D. E. Woon and T. H. Dunning Jr., J. Chem. Phys., 100, 2975 (1994).

[24] K. Ruud, H. Skaane, T. Helgaker, K. L. Bak, and P. Jørgensen, J. Amer. Chem. Soc., 116, 10135 (1994).

[25] K. Ruud, and T. Helgaker. Chem. Phys. Lett., 264, 17 (1997).

[26] T. U. Helgaker, J. Almlöf, H. J. Aa. Jensen, and P. Jørgensen. J. Chem. Phys., 84, 6266 (1986).

[27] T. Helgaker, H. J. Aa. Jensen, P. Jørgensen, J. Olsen, H. Ågren, T. Andersen, K. L. Bak, V. Bakken, O. Christiansen, P. Dahle, E. K. Dalskov, T. Enevoldsen, B. Fernandez, H. Heiberg, H. Hettema, D. Jonsson, S. Kirpekar, R. Kobayashi, H. Koch, K. V. Mikkelsen, P. Norman, M. J. Packer, K. Ruud, T. Saue, P. R. Taylor, and O. Vahtras. DALTON, an $a b$ initio electronic structure program.

[28] S. M. Cybulski and D. M. Bishop, J. Chem. Phys, 100, 2019 (1994).

[29] K. Ruud, T. Helgaker, P. Jørgensen, and K. L. Bak, Chem. Phys. Lett., 223, 12 (1994).

[30] L. Pauling, J. Chem. Phys, 4, 673 (1936).

[31] P. v. R. Schleyer and H. Jiao, Pure and Appl. Chem., 68, 209 (1996). 